\title{
Ressonância magnética para avaliação dos limites dos campos clássicos de radioterapia em pacientes portadoras de neoplasia maligna de colo uterino*
}

\author{
Magnetic resonance imaging in the evaluation of standard radiotherapy field borders \\ in patients with uterine cervix cancer
}

\author{
Geison Moreira Freire ${ }^{1}$, Rodrigo Souza Dias ${ }^{2}$, Adelmo José Giordani ${ }^{3}$, Julisa Chamorro \\ Lascasas Ribalta ${ }^{4}$, Helena Regina Comodo Segreto ${ }^{5}$, Roberto Araujo Segreto ${ }^{6}$
}

Resumo OBJETIVO: Avaliar os limites de campo padronizados para radioterapia de neoplasia maligna de colo uterino com o uso de ressonância magnética e verificar a importância deste exame na redução de possíveis erros de planejamento com técnica convencional. MATERIAIS E MÉTODOS: Foram analisados, retrospectivamente, exames de ressonância magnética do planejamento de 51 pacientes tratadas devido a neoplasia de colo uterino. Os parâmetros estudados foram limites anterior e posterior no corte sagital. RESULTADOS: Observou-se, no corte sagital das ressonâncias magnéticas, que o limite de campo anterior apresentou-se inadequado em 20 $(39,2 \%)$ pacientes e que houve perda geográfica em $37,3 \%$ dos casos no limite posterior. A inadequação de ambos os limites de campo não se relacionou com parâmetros clínicos como idade das pacientes, estadiamento, tipo e grau histológico. CONCLUSÃO: A avaliação dos limites de campo padronizados pela literatura com o uso de ressonância magnética mostrou altos índices de inadequação dos limites do campo lateral, assim como a importância do uso deste exame no planejamento radioterápico de pacientes portadoras de câncer de colo uterino com a finalidade de reduzir a perda geográfica no volume alvo de tratamento.

Unitermos: Câncer de colo uterino; Radioterapia; Imagem por ressonância magnética; Perda geográfica; Volume tumoral.

Abstract OBJECTIVE: To evaluate, by means of magnetic resonance imaging, the standardized field borders in radiotherapy for malignant neoplasm of uterine cervix, and to determine the role of this method in the reduction of possible planning errors related to the conventional technique. MATERIALS AND METHODS: Magnetic resonance imaging studies for planning of treatment of 51 patients with uterine cervix cancer were retrospectively analyzed. The parameters assessed were the anterior and posterior field borders on sagittal section. RESULTS: The anterior field border was inappropriate in 20 (39.2\%) patients and geographic miss was observed in $37.3 \%$ of cases in the posterior border. The inappropriateness of both field borders did not correlate with clinical parameters such as patients' age, tumor staging, histological type and degree. CONCLUSION: The evaluation of standardized field borders with the use of magnetic resonance imaging has demonstrated high indices of inappropriateness of the lateral field borders, as well as the relevant role of magnetic resonance imaging in the radiotherapy planning for patients with uterine cervix cancer with a view to reduce the occurrence of geographic miss of the target volume.

Keywords: Uterine cervix cancer; Radiotherapy planning; Magnetic resonance imaging; Geographic miss; Tumor volume.

Freire GM, Dias RS, Giordani AJ, Ribalta JCL, Segreto HRC, Segreto RA. Ressonância magnética para avaliação dos limites dos campos clássicos de radioterapia em pacientes portadoras de neoplasia maligna de colo uterino. Radiol Bras. 2010;43(3): $175-178$.

* Trabalho realizado no Setor de Radioterapia da Universidade Federal de São Paulo (Unifesp), São Paulo, SP, Brasil.

1. Pós-Graduando Nível de Mestrado do Setor de Radioterapia da Universidade Federal de São Paulo (Unifesp), São Paulo, $\mathrm{SP}$, Brasil.

2. Mestre, Médico Assistente do Setor de Radioterapia da Universidade Federal de São Paulo (Unifesp), São Paulo, SP, Brasil.

3. Doutor, Físico do Setor de Radioterapia da Universidade Federal de São Paulo (Unifesp), São Paulo, SP, Brasil.

4. Livre-Docente, Professora Associada do Departamento de Ginecologia da Universidade Federal de São Paulo (Unifesp), São Paulo, SP, Brasil.

5. Pós-Doutorado, Professora Associada do Setor de Radioterapia da Universidade Federal de São Paulo (Unifesp), São Paulo, SP, Brasil.

\section{INTRODUÇÃO}

O câncer de colo uterino é a segunda neoplasia mais comum entre as mulheres.

6. Livre-Docente, Professor Associado e Chefe do Setor de Radioterapia da Universidade Federal de São Paulo (Unifesp), São Paulo, SP, Brasil.

Endereço para correspondência: Dr. Roberto Araujo Segreto. Rua Pascal, 778, ap. 102, Campo Belo. São Paulo, SP, Brasil, 04616-002. E-mail: segreto.dmed@epm.br

Recebido para publicação em 2/10/2009. Aceito, após revisão, em 16/4/2010.
A maior parte dessas pacientes encontra-se nos países em desenvolvimento, sendo ele considerado o câncer mais incidente no sexo feminino em alguns locais ${ }^{(\mathbf{1})}$. O Instituto Nacional de Câncer (INCA) estimou, para 2010, a ocorrência de 18.430 novos casos desta doença, com um risco estimado de 18 casos a cada 100.000 mulheres ${ }^{(2)}$.

A radioterapia representa importante estratégia terapêutica para esta doença, com finalidade curativa e mesmo paliativa. São 
empregadas duas modalidades de radioterapia no tratamento deste tumor: teleterapia e braquiterapia. A teleterapia, técnica em que a fonte de radiação encontra-se a distância do alvo, é utilizada no tratamento de toda a pelve, incluindo lesão, útero, vagina, paramétrios e drenagens linfáticas. $\mathrm{Na}$ braquiterapia, a fonte de radiação é colocada junto ao volume alvo a ser tratado ${ }^{(3-5)}$.

A técnica utilizada com maior frequência na teleterapia é a de quatro campos, sendo um anterior, outro posterior e dois látero-laterais, incluindo todo o volume alvo a ser tratado ${ }^{(6)}$.

Além dos exames de estadiamento preconizados pela Federação Internacional de Ginecologia e Obstetrícia (FIGO), a ressonância magnética (RM) é de grande valor para pacientes com esta doença, pois permite delineação da anatomia normal da pelve feminina e, principalmente, avaliação da extensão do tumor localmente ${ }^{(7,8)}$.

O objetivo do presente estudo é avaliar os limites de campo padronizados para radioterapia de neoplasia maligna de colo uterino com o uso de RM e verificar a importância deste exame na redução de possíveis erros geográficos com técnica convencional.

\section{MATERIAIS E MÉTODOS}

Antes do início da radioterapia, 51 pacientes foram estadiadas conforme os critérios da FIGO e submetidas ao planejamento, quando são realizadas radiografias ortogonais e simulação dos campos de tratamento, com definição dos limites baseados em referências ósseas padronizadas pela literatura.

Os critérios de inclusão utilizados foram: pacientes com idade $\geq 21$ anos, diagnóstico de neoplasia de colo uterino confirmado com anatomopatológico, pacientes em estádio clínico IB a IVA (FIGO) e realização de RM pré-tratamento. Os critérios de exclusão foram: contraindicação para RM, realização de tratamento prévio e presença de outra neoplasia em pelve concomitante ou anteriormente tratada.

No planejamento radioterápico clássico utilizam-se como limites para os campos anterior e posterior a transição da quarta para quinta vértebras lombares, a borda inferior do forame obturador ou da pube, dependendo do acometimento vaginal, e lateralmente, a 1,5 cm de margem da pequena pelve. Nos campos laterais, o limite anterior (LA) é a sínfise púbica (borda anterior) e o limite posterior (LP), a transição entre a segunda e terceira vértebras sacrais (S2-S3) ou incluindo toda a vértebra $S 3^{(4)}$. Todas as pacientes realizaram RM de pelve antes do início do tratamento. Foram feitas sequências ponderadas em T1 e T2 com cortes sagitais, em aparelho da marca Philips de 1,0 tesla.

Com auxílio de um radiologista, os volumes de tratamento foram delineados nos exames de RM, considerando como volume alvo o tumor e o corpo uterino, e foi escolhido o corte que apresentava o volume com maior diâmetro ântero-posterior. As RMs foram analisadas retrospectivamente quanto à inclusão do volume alvo nos campos padronizados da radioterapia. Os parâmetros estudados foram os limites anterior e posterior no corte sagital ao nível da linha mediana. Foi empregada margem de segurança de $1,0 \mathrm{~cm}$ em torno do tumor e corpo uterino.

Os dados obtidos foram submetidos a análise estatística. A análise inferencial empregada com o intuito de confirmar ou refutar evidências encontradas na análise descritiva foi a regressão logística. $\mathrm{O}$ valor de significância para rejeição da hipótese de nulidade foi $p \leq 0,05$.

As características clínicas das pacientes, como idade, tipo histológico, grau histológico e estadiamento FIGO, estão apresentados na Tabela 1 .

\section{RESULTADOS}

A avaliação da adequação dos limites de campo no corte sagital das RMs mostrou LA inadequado em 20 (39,2\%) das pacientes e perda geográfica em $37,3 \%$ dos casos no LP (Figura 1).

Não foi observada relação entre a inadequação do LA do campo lateral com a idade $(p=0,970)$, estadiamento $(p>$ $0,999)$, tipo $(p=0,162)$ e grau histológico $(p=0,884)$. Da mesma forma, a inadequação do LP não se relacionou com os parâmetros clínicos idade $(p=0,726)$, estadiamento $(p>0,999)$, tipo $(p=0,496)$ e grau histológico $(p=0,813)$.

Em relação ao estadiamento, observouse alta porcentagem de inadequação nos es-

Tabela 1 Características clínicas das pacientes da amostra.

\begin{tabular}{lccc}
\hline Características da amostra & & $n$ & Porcentagem \\
\hline Idade (mediana em anos) & 54 & - & - \\
Tipo histológico & Carcinoma espinocelular & 45 & $88,2 \%$ \\
& Adenocarcinoma & 6 & $11,8 \%$ \\
Grau histológico & $X^{*}$ & 10 & $19,6 \%$ \\
& 1 & 3 & $5,9 \%$ \\
& 2 & 25 & $49,0 \%$ \\
Estadiamento (FIGO) & 3 & 13 & $25,5 \%$ \\
& IB & 3 & $5,9 \%$ \\
& IIB & 29 & $56,9 \%$ \\
\end{tabular}

* Grau X: grau histológico não referido pelo patologista.

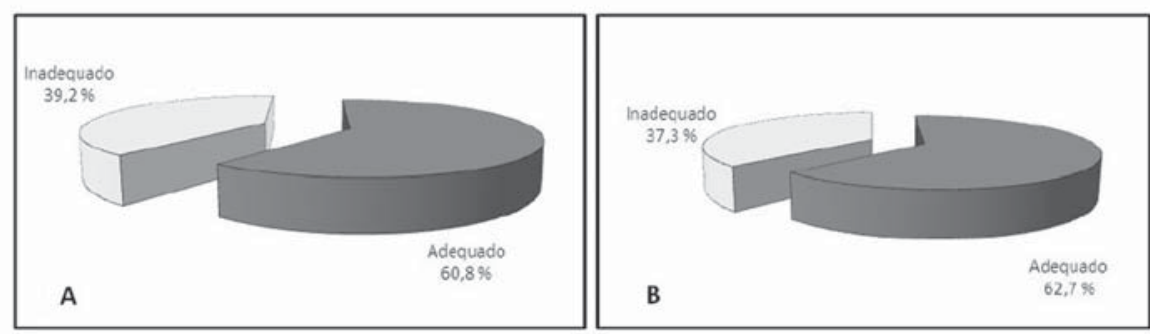

Figura 1. Distribuição dos indivíduos, segundo a adequação dos limites anterior $(\mathbf{A})$ e posterior (B) do campo lateral. 
Figura 2. Distribuição do estadiamento dos indivíduos, segundo a adequação dos limites anterior $(\mathbf{A})$ e posterior (B) do campo lateral (por estádio).
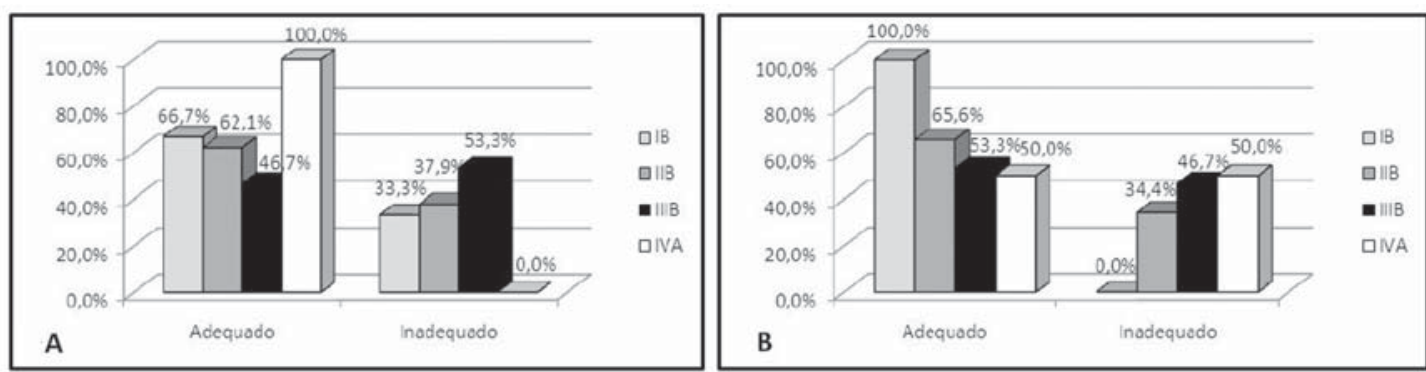

tádios IIB (LA, 37,9\% e LP, 34,4\%) e IIIB (LA, 53,3\% e LP, 46,6\%), analisando pacientes em cada estádio específico. Estes resultados demonstram que não houve significância estatística com os parâmetros analisados e, consequentemente, podem ocorrer erros em qualquer estadiamento ou grau histológico (Figura 2).

$\mathrm{Na}$ análise da adequação de cada grau histológico, encontrou-se inadequação de $33,3 \%$ (LA) no grau 1, 36\% (LA) e $40 \%$ (LP) no grau 2, e 46,1\% (LA) e 46,2\% (LP) no grau 3, mostrando também um maior erro de planejamento em graus histológicos maiores.

\section{DISCUSSÃO}

A radioterapia constitui a principal modalidade terapêutica para tumores de colo de útero localmente avançados, e o planejamento radioterápico é fundamental para que o tratamento seja administrado de forma adequada. Na definição dos limites dos campos de radioterapia, classicamente, eram empregadas referências ósseas para delimitá-los de forma padronizada, não considerando características tumorais e individuais dos pacientes. No presente estudo, foram avaliados os limites anterior e posterior do campo lateral com o uso da RM, para verificar a adequação dos campos planejados.

Nossos resultados referentes às características das pacientes mostram idade mediana de diagnóstico da doença de 54 anos (22 a 77 anos), com predominância do carcinoma espinocelular em $88,2 \%$ dos casos e estadiamento localmente avançado (94,1\%). Este tipo histológico é relatado como o mais frequente na literatura ${ }^{(\mathbf{9 - 1 1})}$. Os tumores mais indiferenciados grau $2(49 \%)$ e grau $3(25,5 \%)$ foram predominantes. A idade mediana observada foi semelhante à encontrada em outros estudos com pacien- tes portadoras da mesma doença ${ }^{(\mathbf{9 , 1 1})}$. Dessa forma, nossos dados corroboram os da literatura, exceto os relacionados aos graus histológicos predominantes, pois os estudos que avaliaram o uso da RM no planejamento de radioterapia não fazem referência a este parâmetro ${ }^{(7,12,13)}$.

Quando avaliada a adequação dos limites de campo lateral, foi observada grande frequência de inadequação, tanto no LA $(39,2 \%)$ quanto no LP $(37,3 \%)$. Em estudo em que foi analisado o corte sagital de 25 RMs de pacientes portadoras de neoplasia de colo uterino, foi observado tumor além do LP em seis pacientes, mostrando a importância da imagem para a avaliação dos limites do campo lateral ${ }^{(\mathbf{1 2})}$.

Zunino et al. aplicaram o mesmo método de análise de RM, utilizando $1 \mathrm{~cm}$ de margem do volume tumoral ou do fundo uterino e encontraram inadequação no LP em $49 \%$ e no LA em $8,8 \%$ dos $\operatorname{casos}^{(7)}$.

Em estudo nacional foram observados índices de inadequação de $46 \%$ (LA) e $40 \%$ $(\mathrm{LP})^{(14)}$, semelhantes aos encontrados no presente estudo. $\mathrm{O}$ mesmo grupo apresenta a atualização destes dados com 80 pacientes, sendo encontrados $36 \%$ de perda geográfica no LA e $35 \%$ no LP. No total, $56 \%$ das pacientes apresentavam algum tipo de inadequação. Nesse estudo foi observada relação significante de inadequação dos limites de campo com diâmetro tumoral ântero-posterior, deslocamento da parede anterior do reto e volume tumoral. Os autores sugerem que estes fatores podem ser usados como preditores de risco de perda geográfica $^{(\mathbf{1 3})}$.

Em relação ao estadiamento, de acordo com a classificação da FIGO, não se observou influência significante do mesmo na adequação ou inadequação dos limites de campo de tratamento, talvez devido à falta de exames de imagem, como tomografia computadorizada (TC) e RM, neste sistema de estadiamento ${ }^{(\mathbf{1 5})}$. Todavia, o estadiamento da FIGO permite uma padronização da avaliação pré-tratamento de pacientes em diferentes regiões do mundo, mesmo em locais onde o acesso a exames de maior complexidade é difícil, para comparação de resultados terapêuticos.

Outro estudo utilizou o sistema de estadiamento TNM para avaliação da adequação dos limites de campo, que permitiu a avaliação do comprometimento linfonodal. Porém, não foi realizada análise estatística devido ao reduzido número de pacientes, sendo feita apenas análise descritiva, que mostrou grande número de pacientes com estadiamento T2B e com ausência de linfonodos comprometidos ${ }^{(16)}$.

Em relação à análise de cada estádio específico, observou-se alta porcentagem de inadequação em estádios IIB (LA, $37,9 \%$ e LP, 34,4\%) e IIIB (LA, 53,3\% e LP, $46,6 \%$ ), dado este que não apresentou significância estatística. Na literatura, Zunino et al. encontraram inadequação, de acordo com o estadiamento, nos estádios IB (LA, 0\% e LP, 50\%), IIA (LA, 0\% e LP, 67\%), IIB (LA, 5\% e LP, 42\%), IIIB (LA, $67 \%$ e LP, $33 \%$ ) e IVA (LA, 0\% e LP, $100 \%)^{(7)}$.

Dessa forma, os dados obtidos no presente estudo mostram maiores erros geográficos quanto maior o estádio, e talvez um aumento da amostra possa evidenciar estatisticamente esse dado.

Com relação ao grau histológico, que representa a diferenciação tumoral e constitui fator importante para avaliação da doença pré-tratamento, este parâmetro mostrou porcentagem de inadequação de $33,3 \%$ (LA) e $0 \%$ (LP) no grau $1,36 \%$ (LA) e $40 \%$ (LP) no grau 2 e 46,1\% (LA) e $46,1 \%$ (LP) no grau 3. Estes resultados também demonstram maior erro de planejamento em graus histológicos maiores, embora não tenham sido significantes. Este achado pode estar correlacionado ao fato 
de graus tumorais maiores estarem associados a tumores mais avançados ou mais volumosos. Na literatura consultada a respeito da inadequação de limites de campo com o uso de RM, os autores não avaliaram este fator em relação à perda geográfica $^{(7,12,13)}$

Na avaliação pré-tratamento, exames de imagem como a TC ou RM estariam indicados para definição do diâmetro e volume tumoral, avaliação de linfonodos pélvicos e invasão parametrial ${ }^{(\mathbf{1 7 - 2 0})}$. Meta-análise realizada com 57 estudos mostrou maior sensibilidade da RM, comparada à TC, na avaliação de invasão parametrial ${ }^{(21)}$. No entanto, em um estudo multicêntrico prospectivo com 208 pacientes portadoras de neoplasia de colo uterino submetidas à avaliação clínica pela FIGO, TC helicoidal e RM, e comparados aos achados cirúrgicos (patológicos), observou-se que a TC e a RM apresentaram resultados semelhantes na avaliação de invasão parametrial e concluiu-se que o exame físico era superior a outros exames. Os autores sugeriram influência dos exames de imagem no estadiamento clínico e que tais exames poderiam aprimorar o estadiamento das pacientes antes do tratamento ${ }^{(22)}$.

Estudo recente mostra a importância da RM como fator prognóstico para analisar volume tumoral e invasão uterina pelo tumor, e TC para avaliar linfonodos pélvicos $\operatorname{positivos}^{(23)}$.

$\mathrm{O}$ mais recente guideline do National Comprehensive Cancer Network (NCCN) sugere o uso de TC ou RM para definição do planejamento terapêutico, porém estes exames não interferem no estadiamento formal, sendo o estadiamento da FIGO considerado padrão para comparação entre tratamentos ${ }^{(24)}$.

Dessa forma, embora o custo do uso da RM para planejamento com radioterapia rotineiramente seja um dos principais fatores que dificultam a implementação deste exame em países pobres ou em desenvolvimento, nossos resultados, juntamente com a revisão da literatura realizada, sugerem que a RM constitui exame de grande importância para o planejamento radioterápico de pacientes portadoras de colo uterino, e este deve ser individualizado considerando variações anatômicas e de volume tumoral ${ }^{(7,12,13,16)}$. A neoplasia de colo uterino assume grande importância como a terceira causa de morte em mulheres em todo o mundo, provocando em torno de 274.000 óbitos anuais, sendo $78 \%$ em países em desenvolvimento ${ }^{(\mathbf{1})}$, e a RM pode trazer benefício para otimização do tratamento dessas pacientes.

\section{CONCLUSÕES}

Analisados em conjunto, os dados obtidos no presente estudo mostraram que a RM é uma ferramenta importante para o planejamento radioterápico de pacientes portadoras de câncer de colo uterino, pois permite maior acurácia na definição dos limites de campos de irradiação, reduzindo, assim, os erros geográficos. A idade, o estadiamento, o grau e o tipo histológico não se relacionaram com a inadequação dos campos, conforme estudado.

\section{REFERÊNCIAS}

1. Parkin DM, Bray F, Ferlay J, et al. Global cancer statistics, 2002. CA Cancer J Clin. 2005;55:74108.

2. Brasil. Ministério da Saúde. Instituto Nacional de Câncer. Estimativa 2010: incidência de câncer no Brasil. Rio de Janeiro, RJ: Instituto Nacional de Câncer; 2009

3. Perez CA, Kavanagh BD. Uterine cervix. In: Perez CA, Brady LW, Halperin EC, et al., editors. Principles and practice of radiation oncology. 4th ed. Philadelphia, PA: Lippincott-Raven; 2004.

4. Oliveira JP, Rosa LAR, Batista DVS, et al. Avaliação da dose no reto em pacientes submetidas a braquiterapia de alta taxa de dose para o tratamento do câncer do colo uterino. Radiol Bras. 2009;42: 83-8.

5. Guimarães RGR, Carvalho HA, Stuart SR, et al. Avaliação dosimétrica de uma combinação de aplicadores para braquiterapia de tumores do colo uterino com acometimento da porção distal da vagina. Radiol Bras. 2009;42:209-14.

6. Swift PS, Hsu CJ. Cancer of the uterine cervix. In: Leibel SA, Philips TL, editors. Textbook of radiation oncology. 2nd ed. Philadelphia, PA: Saunders; 2004

7. Zunino S, Rosato O, Lucino S, et al. Anatomic study of the pelvis in carcinoma of the uterine cervix as related to the box technique. Int J Radiat Oncol Biol Phys. 1999;44:53-9.

8. Mayr NA, Tali ET, Yuh WT, et al. Cervical cancer: application of MR imaging in radiation therapy. Radiology. 1993;189:601-8.

9. Thomas G, Ali S, Hoebers FJ, et al. Phase III trial to evaluate the efficacy of maintaining hemoglobin levels above $12.0 \mathrm{~g} / \mathrm{dL}$ with erythropoietin vs above $10.0 \mathrm{~g} / \mathrm{dL}$ without erythropoietin in anemic patients receiving concurrent radiation and cisplatin for cervical cancer. Gynecol Oncol. 2008;108:317-25.

10. Torres MA, Jhingran A, Thames HD Jr, et al. Comparison of treatment tolerance and outcomes in patients with cervical cancer treated with concurrent chemoradiotherapy in a prospective randomized trial or with standard treatment. Int $J$ Radiat Oncol Biol Phys. 2008;70:118-25.

11. Huguet F, Cojocariu OM, Levy P, et al. Preoperative concurrent radiation therapy and chemotherapy for bulky stage IB2, IIA, and IIB carcinoma of the uterine cervix with proximal parametrial invasion. Int J Radiat Oncol Biol Phys. 2008;72:1508-15

12. Russell AH, Walter JP, Anderson MW, et al. Sagittal magnetic resonance imaging in the design of lateral radiation treatment portals for patients with locally advanced squamous cancer of the cervix. Int J Radiat Oncol Biol Phys. 1992;23:449-55.

13. Justino PB, Baroni R, Blasbalg R, et al. Clinical tumor dimensions may be useful to prevent geographic miss in conventional radiotherapy of uterine cervix cancer - a magnetic resonance imaging-based study. Int J Radiat Oncol Biol Phys 2009;74:503-10.

14. Justino PB, Carvalho HA, Baroni R, et al. Valor da ressonância magnética no planejamento radioterápico dos tumores de colo de útero: resultados preliminares. Radiol Bras. 2005;38:399-402.

15. Creasman WT. New gynecologic cancer staging. Gynecol Oncol. 1995;58:157-8.

16. Thomas L, Chacon B, Kind M, et al. Magnetic resonance imaging in the treatment planning of radiation therapy in carcinoma of the cervix treated with the four-field pelvic technique. Int J Radiat Oncol Biol Phys. 1997;37:827-32.

17. Greco A, Mason P, Leung AW, et al. Staging of carcinoma of the uterine cervix: MRI-surgical correlation. Clin Radiol. 1989;40:401-5.

18. Hawnaur JM, Johnson RJ, Carrington BM, et al Predictive value of clinical examination, transrectal ultrasound and magnetic resonance imaging prior to radiotherapy in carcinoma of the cervix. Br J Radiol. 1998;71:819-27.

19. Toita T, Kakinohana Y, Shinzato S, et al. Tumor diameter/volume and pelvic node status assessed by magnetic resonance imaging (MRI) for uterine cervical cancer treated with irradiation. Int J Radiat Oncol Biol Phys. 1999;43:777-82.

20. Wagenaar HC, Trimbos JB, Postema S, et al. Tumor diameter and volume assessed by magnetic resonance imaging in the prediction of outcome for invasive cervical cancer. Gynecol Oncol. 2001;82:474-82.

21. Bipat S, Glas AS, van der Velden J, et al. Computed tomography and magnetic resonance imaging in staging of uterine cervical carcinoma: a systematic review. Gynecol Oncol. 2003;91:59_ 66

22. Hricak H, Gatsonis C, Chi DS, et al. Role of imaging in pretreatment evaluation of early invasive cervical cancer: results of the intergroup study American College of Radiology Imaging Network 6651-Gynecologic Oncology Group 183. J Clin Oncol. 2005;23:9329-37

23. Kim H, Kim W, Lee M, et al. Tumor volume and uterine body invasion assessed by MRI for prediction of outcome in cervical carcinoma treated with concurrent chemotherapy and radiotherapy. Jpn J Clin Oncol. 2007;37:858-66.

24. The NCCN Cervical Cancer Guideline, Version 1.2009. In: The Complete Library of NCCN Clinical Practice Guidelines in Oncology, 2009 [database on the Internet]. 